\title{
Bounds on neutrino-scalar nonstandard interactions from big bang nucleosynthesis
}

\author{
Jorge Venzor $\odot^{*}$ and Abdel Pérez-Lorenzana $\odot^{\dagger}$ \\ Departamento de Física, Centro de Investigación y de Estudios Avanzados del I.P.N., \\ Apartado Postal 14-740, 07000 Ciudad de México, México \\ Josue De-Santiago $\oplus^{\ddagger}$ \\ Departamento de Física, Centro de Investigación y de Estudios Avanzados del I.P.N., \\ Apartado Postal 14-740, 07000 Ciudad de México, México \\ and Cátedra-Consejo Nacional de Ciencia y Tecnología, Avenida Insurgentes Sur 1582, \\ 03940 Ciudad de México, México
}

(Received 16 September 2020; revised 4 February 2021; accepted 8 February 2021; published 25 February 2021)

Coherent forward scattering processes by neutrino-scalar nonstandard interactions (SNSI) induce an effective neutrino mass. In the early Universe, a large neutrino effective mass restricts the production of neutrinos. The SNSI effect is modulated by two effective couplings; these account for the coupling between neutrinos and electrons or positrons, $G_{\text {eff }}$, and the neutrino self-interaction $G_{\mathrm{S}}$. These parameters are directly related to the effective number of relativistic species, and nonzero values imply a smaller than expected $N_{\text {eff }}$. We employ big bang nucleosynthesis to constraint the SNSI effect. We find that $G_{\text {eff }}<$ $1.2 \mathrm{MeV}^{-2}$ and $G_{\mathrm{S}}<2.0 \times 10^{7} \mathrm{MeV}^{-2}$ at $68 \%$ C.L. For a scalar mass in the range $10^{-15} \mathrm{eV} \lesssim m_{\phi} \lesssim 10^{-5} \mathrm{eV}$, our neutrino-scalar coupling constraint is more restrictive than any previous result.

DOI: 10.1103/PhysRevD.103.043534

\section{INTRODUCTION}

Despite being light, neutrino gravitational interaction plays an essential role in shaping the distribution of matter and energy in the Universe. Several cosmological surveys have led to the strongest bounds on the sum of the neutrino masses [1-4]. These are one order of magnitude better than those from experimental counterparts [5]. Cosmology now leads the race to determine the neutrino mass hierarchy and, possibly, measure the mass of at least one neutrino throughout this decade [6,7]. Moreover, three standard neutrinos are required to predict accurately the abundance of light elements on the Universe through big bang nucleosynthesis $(\mathrm{BBN})$ [8-10]. This is in concordance with the standard precision computation of the neutrino contribution to radiation density, that can be expressed in terms of the parameter $N_{\text {eff }} \simeq 3.046$ [11-15].

*jorge.venzor@cinvestav.mx

aplorenz@fis.cinvestav.mx

¥Josue.desantiago@cinvestav.mx

Published by the American Physical Society under the terms of the Creative Commons Attribution 4.0 International license. Further distribution of this work must maintain attribution to the author(s) and the published article's title, journal citation, and DOI. Funded by SCOAP.
Cosmological model-independent bounds on neutrinos will be more reliable by disentangling the effects of neutrino parameters with the rest of cosmological ones [1]. As an important step, the existence of relativistic species in the early Universe has been proven by detecting a phase shift on the acoustic oscillations that cannot be mimicked by other cosmological parameters [16-18]. In this sense, cosmology has become a fruitful lab to test neutrino physics in the outline of the standard model of particle physics (SM) and beyond (BSM).

Neutrino interactions with matter are crucial to study them. For instance, the Mikheyev-Smirnov-Wolfenstein effect [19], which changes the neutrino oscillations in matter, was used to determine the sign of the square-mass splitting $\Delta m_{21}^{2}>0$ in the solar neutrino experiments (see, for instance, [20,21]). The same mechanism is being brought out by long-baseline neutrino experiments aiming to determine the sign of $\Delta m_{31}^{2}$ (see experiments in Refs. [22,23]).

In cosmology, a neutrino nonstandard interaction (NSI) may solve some tensions in the standard theory. It has been studied whether an NSI may explain the discrepancy known as the $H_{0}$ tension, where the measurement of $H_{0}$ by the cosmic microwave background (CMB) and local observations are clearly in statistical disagreement [1,24-26]. There are some approaches that try to solve this problem using NSI, including interactions in the sterile [27] 
or in the active neutrino sectors [28]. In the latter approach, neutrinos are required to be either strongly self-interacting $(\mathrm{SI} \nu)$ or moderately self-interacting $(\mathrm{MI} \nu)$.

$\mathrm{SI} \nu$ and/or MI $\nu$ are assumed to be mediated by a scalar particle with a mass larger than $\mathcal{O}(\mathrm{keV})$. And the parameter space, in this approximation, has been cornered by experimental, astrophysical, and BBN constraints [29-33]. On the other hand, the phenomenology of neutrino-scalar nonstandard interactions (SNSI) mediated by a light particle is rich and has several consequences. For instance, large-scale structure (LSS) data constrain neutrino dispersion mediated by a scalar much lighter than $\mathcal{O}(\mathrm{eV})$ [34,35]. Furthermore, neutrinos may annihilate and decay into lighter bosons, which, interestingly, may relax the bound on $\sum m_{\nu}$ imposed by LSS [36-40].

Although the information on the light mediator mass is lost when studying two-body dispersion in the regime $m_{\phi} \ll T_{\nu}$, loop diagrams such as mass-correction type, a priori, are mass dependent regardless of the smallness of the scalar mass. Therefore, studying this kind of diagrams within the early Universe background is convenient if we are to search for mediator mass-dependent SNSI constraints.

In this manuscript, we explore the cosmological consequences of neutrino SNSI mass-correction processes mediated by a light scalar particle $\left(10^{-15} \mathrm{eV} \lesssim m_{\phi} \lesssim 10^{-5} \mathrm{eV}\right)$. We assess the calculations performed by Babu, Chauhan, and Dev [41] in the early Universe. Mass-correction diagrams involving an SNSI have received recent attention because Ge and Parke [42] found a small solar neutrino data preference for nonvanishing SNSI couplings with ordinary matter. This result has led to further research about neutrino propagation with SNSI in Earth, the Sun, and supernovae $[41,43,44]$.

For our exploration, we identify two effective parameters that modulate the SNSI effect and study its consequences. We solve numerically the mass contribution and the evolution of the neutrino density. Additionally, we notice that large effective SNSI couplings may noticeably change the neutrino contribution to radiation. This information is encoded through a temperature-dependent change on the effective number of relativistic species $N_{\text {eff }}$. In order to find $N_{\text {eff }}$, we employ a modified version of the public code NUDEC_BSM $[13,45]$. A change on $N_{\text {eff }}$ straightforwardly alters the expansion rate during the radiation-dominated era, affecting the proton $\leftrightarrow$ neutron freeze-out temperature and, hence, the neutron-to-proton ratio right at the unset of BBN. Thus, the production of primordial nuclei helps us to constrain the SNSI parameter space. We use a modified version of the public BBN code ALTERBBN [46,47] to find the parameter constraints. Finally, we translate these bounds into the scalar mass-couplings parameter space and compare them with other results.

The rest of the paper is organized as follows. In Sec. II, we review and discuss the properties of the effective mass coherent forward scattering process (CFS) by SNSI at high temperatures. In Sec. III, we explore the phenomenological consequences of the effective neutrino mass. Then, in Sec. IV, we constrain the parameter space of the SNSI with BBN theory and the abundance of light elements. In Sec. V, we compare our constraints with laboratory, astrophysical, and cosmological bounds on the parameters. Our conclusions are summarized in Sec. VI.

\section{NEUTRINO-SCALAR NONSTANDARD INTERACTIONS}

The outcomes of neutrino NSI depend on the nature of the mediator particle. On the one hand, vector-mediated NSI has a phenomenology that produces similar effects as the weak interaction. The SNSI, instead, appears as a Yukawa term on the effective Lagrangian [42] which induces an effective mass. This mass term depends on the properties of the environment where neutrinos propagate. A dense and hot background may produce a large neutrino mass.

We are interested in the effect of the SNSI in the CFS described by the tadpole diagram in Fig. 1. We consider that neutrinos are propagating in a hot plasma when the Universe had a temperature around some MeVs; this plasma is composed of photons, baryons, charged leptons, and the three standard neutrinos. The SNSI effect in the neutrino propagation can be interpreted as a refractive index $[48,49]$. Here, we focus on a generic scalar interaction, ignoring the details of an underlying particle physics model, having the cosmological phenomenology as our main approach.

The effective neutrino mass described by the quantum correction would be

$m_{\text {eff }}=m_{\nu}+2 G_{\text {eff }} \Delta m\left(m_{e} ; T_{\gamma}\right)+3 G_{\mathrm{S}} \Delta m\left(m_{\nu} ; T_{\nu}\right)$,

where $m_{\nu}$ is the bare neutrino mass, and the correction is described by [41]

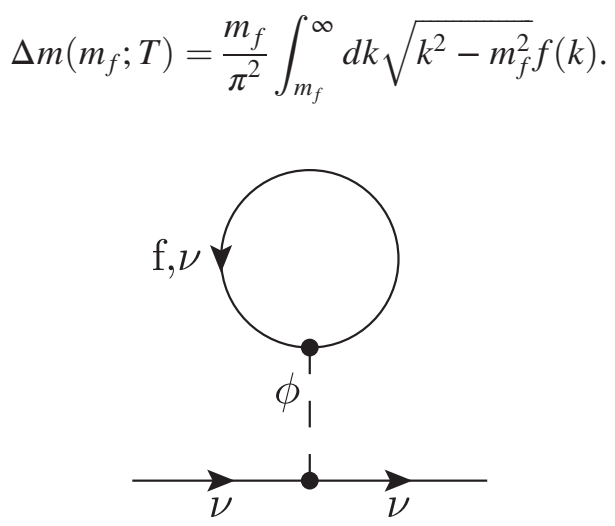

FIG. 1. Tadpole diagram of the neutrino CFS with a background of leptons. This process induces a thermal correction to neutrino mass. 
Here $m_{f}$ is the mass of the fermion and $f\left(k_{0}\right)$ is the FermiDirac distribution for the the background fermions. Safely neglecting the chemical potential [50] $\mu=0$, the FermiDirac distribution for both fermions and antifermions is the same $\left(\mathrm{e}^{k / T}+1\right)^{-1}$, where $T$ is the temperature of the thermal background. The two free parameters $G_{\text {eff }}$ and $G_{\mathrm{S}}$ are then given as

$$
G_{\text {eff }}=\frac{g_{f} g_{\nu}}{m_{\phi}^{2}}
$$

and

$$
G_{\mathrm{S}}=\frac{g_{\nu}^{2}}{m_{\phi}^{2}}
$$

respectively, where $m_{\phi}$ is the the mass of the scalar mediator, $g_{\nu}$ is the neutrino-scalar coupling, and $g_{f}$ is the coupling between the scalar and charged leptons. These effective couplings encode the strength of the interaction and are the ones to be constrained by observations. Here we assume universal couplings with both charged lepton and neutrino flavors. Therefore, all complex phases can be absorbed, and one can assume neutrino mass corrections to be always positive. Notice that, at the temperatures that we are interested here, there are not muons or taus present in the plasma, since they have already decayed into lighter particles by then $\left(T_{\gamma} \ll m_{\mu} \sim 105.65 \mathrm{MeV}\right)$. Hence, we take into account only couplings with electrons and positrons.

The numerical solution of the electron or positron SNSI contributing to the neutrino mass is depicted in Fig. 2.

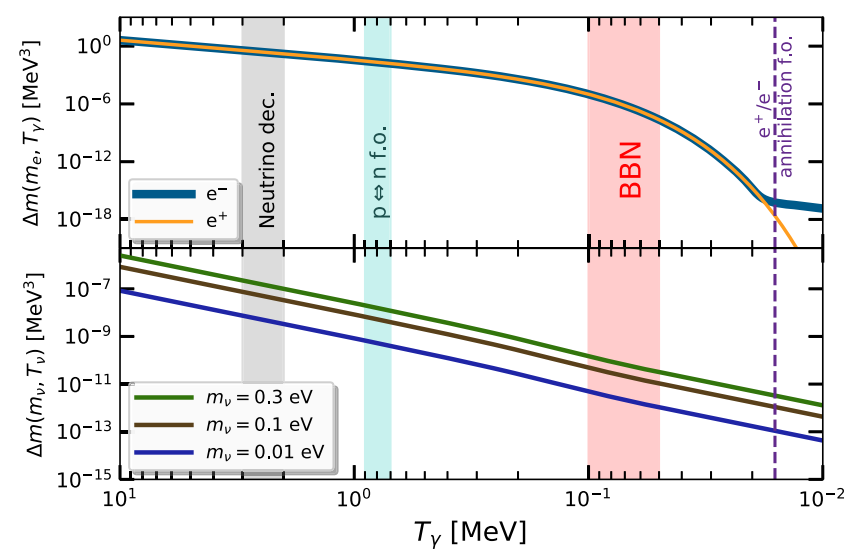

FIG. 2. Neutrino mass correction induced by an SNSI interaction, depicted as a function of the photon-baryon temperature. Upper panel: SNSI with electrons and positrons. Lower panel: neutrino self-interaction for three different values of the neutrino bare mass present in the background. Key cosmological events highlighted: neutrino decoupling, proton to neutron freeze-out (f.o.), synthesis of light elements, and electron-positron annihilation freeze-out.
At high temperatures, both contributions to the effective mass are the same. Below the electron mass threshold, the contribution decays exponentially as the Universe cools down. But, when the electron-positron annihilation ends, only electrons remain in the background. However, at temperatures much smaller than $\mathcal{O} \mathrm{MeV}$, the neutrino mass-correction contribution induced by leptons becomes negligible.

Unlike some terrestrial and astrophysical scenarios, here we also need to consider the background composed of relic neutrinos. In this self-interacting case, $\Delta m$ would have another unknown parameter, the bare neutrino mass $m_{\nu}$. Notice that, in order to have a $\Delta m$ of the same order of magnitude as the one induced by charged leptons, $G_{\mathrm{S}}$ needs to be roughly $m_{\mathrm{e}} / m_{\nu}$ times larger than $G_{\text {eff }}$; see Eq. (2). As the BBN epoch occurs at temperatures much larger than the bare neutrino mass scales, the mass correction does not drop exponentially with the temperature as occurs with the electron or positron SNSI. By definition, by constraining $G_{\mathrm{S}}$, we would be able to find a mediator mass-dependent $g_{\nu}$ bound.

By oscillation experiments, we know that at least two neutrinos are massive. Hereafter, we shall take a conservative value for bare neutrino masses, being onethird of the minimum sum of neutrino masses in the normal hierarchy, $\left(\sum m_{\nu}\right)_{\min } \sim 0.059 \mathrm{eV}$ [3], assuming an almost degenerate scenario of active neutrinos. Given this, we take $m_{\nu}=0.0195 \mathrm{eV}$ and assume all three neutrino parameters are universal.

\section{COSMOLOGICAL IMPLICATIONS}

In the previous section, we have described how the neutrino $m_{\text {eff }}$ would be affected by CFS with charged leptons and neutrinos at high temperatures. We now focus on the implementation and implications of neutrino SNSI in the early Universe. In particular, in this section, we compute $N_{\text {eff }}$ as a function of the SNSI parameters.

The particles in the plasma are in local thermal equilibrium when their interaction rate is larger than the rate of the expansion of the Universe, $\Gamma \gg H\left(T_{\gamma}\right)$. The Universe at high temperatures $\left(T_{\gamma} \sim \mathcal{O} \mathrm{MeV}\right)$ is dominated by radiation, and the density of any heavy particle, $m \gtrsim T_{\gamma}$, gets suppressed. A large neutrino $m_{\text {eff }}$ will diminish its production by weak interactions, and, ultimately, the Universe will have less radiation than expected. Therefore, by weighting the effect of $m_{\text {eff }}$ on $N_{\text {eff }}$, we will estimate the permitted values of the SNSI parameters.

As we stated in the previous section, the mass-correction diagram in Fig. 1 is describing a CFS that implies no transfer of energy and momentum with the plasma. Therefore, a priori, the neutrino thermal evolution should remain unchanged. Nonetheless, we carefully explore whether the $m_{\text {eff }}$ is capable of changing the thermal evolution of neutrinos. 
In this scenario, the weak interaction is the one responsible for keeping neutrinos in thermal equilibrium with the plasma. In equilibrium, the neutrino energy and number density, for one flavor, are given, respectively, by [51]

$$
\begin{aligned}
& \rho_{\nu}\left(G_{\mathrm{eff}}, G_{\mathrm{S}} ; T_{\nu}, T_{\gamma}\right)=\frac{T_{\nu}^{4}}{\pi^{2}} \int_{\alpha}^{\infty} \frac{d x x^{2} \sqrt{x^{2}-\alpha^{2}}}{e^{x}+1}, \\
& n_{\nu}\left(G_{\mathrm{eff}}, G_{\mathrm{S}} ; T_{\nu}, T_{\gamma}\right)=\frac{T_{\nu}^{3}}{\pi^{2}} \int_{\alpha}^{\infty} \frac{d x x \sqrt{x^{2}-\alpha^{2}}}{e^{x}+1}
\end{aligned}
$$

where $x=E_{\nu} / T_{\nu}$ and $\alpha=m_{\mathrm{eff}} / T_{\nu}$. The effective mass $m_{\text {eff }}$ encodes all the new physics, as given in Eq. (1). Notice that the neutrino density gets suppressed with a larger $m_{\text {eff }}$. As the Universe cools down, the effective neutrino mass drops significantly; this permits the neutrino density to approach and possibly recover its standard value. However, after neutrino decoupling, it is not possible to produce abundantly new neutrinos to reach their standard density. Thus, establishing the neutrino decoupling temperature is important to compute the final neutrino density to a good approximation.

The neutrino thermal mass, if relevant, would increase the temperature at which neutrinos decouple. We compare the interaction rate of electron-neutrino scattering, which is the responsible to keep neutrinos in equilibrium, with the expansion rate. In the standard theory, we have $\Gamma_{e w} \propto\left(1-m_{e}^{2} / T^{2}\right)^{2} T^{5}$, while the expansion rate is proportional to the energy density $H(T) \propto \sqrt{\rho}$. In the radiationdominated epoch $\rho \propto T^{4}$, and, thus, $H \propto T^{2}$. The SNSI effect diminishes both the interaction and the expansion rates, although we have found that the dominant effect comes from the interaction rate depletion. We estimate the ratio of the cross section to the SM one to be [52]

$\frac{\sigma}{\sigma_{\mathrm{SM}}}=\frac{2 \sqrt{A_{+} A_{-}}}{3\left(1-m_{e}^{2} / T^{2}\right)^{2}}\left[A_{+} A_{-}+1-\frac{A_{+}+A_{-}}{4}-B_{-}^{2}\right]$,

where $A_{+}=1-\left(\left(m_{e}+m_{\mathrm{eff}}\right) / T\right)^{2}, A_{-}=1-\left(\left(m_{e}-m_{\mathrm{eff}}\right) / T\right)^{2}$, $B_{-}=\left(m_{e}^{2}-m_{\mathrm{eff}}^{2}\right) / T^{2}$, and the condition $m_{e}+m_{\mathrm{eff}}<T$, which is true for the permitted parameter region we will present in the next section. Note that in the limit $m_{\text {eff }} \rightarrow 0$ we have $\sigma / \sigma_{\mathrm{SM}}=1$.

We solve numerically the equation $\Gamma=H$ for each pair $\left(G_{\mathrm{S}}, G_{\text {eff }}\right)$ to find the decoupling temperature due to SNSI. We model the interaction rate as $\Gamma=\left\langle\sigma v>n_{e}=\xi \sigma n_{e}\right.$, where $\xi$ encodes our ignorance about the thermal average, $n_{e}=3 \zeta(3) T^{3} /\left(2 \pi^{2}\right)$ is the electron or positron density, and

$\sigma=\frac{2}{3 \pi} G_{\mathrm{F}}^{2} T^{2} \sqrt{A_{+} A_{-}}\left[A_{+} A_{-}+1-\frac{A_{+}+A_{-}}{4}-B_{-}^{2}\right]$,

where $G_{\mathrm{F}} \sim 1.166 \times 10^{-11} \mathrm{MeV}^{-2}$ is the Fermi constant. We set the value of $\xi \sim 6.5$ to match the most conservative value for the standard case $T_{\mathrm{dec} \text { std }}=2 \mathrm{MeV}$. Furthermore, we assume the value of $\xi$ does not change due to SNSI. Interestingly, we find a region of the parameter space $\left(G s>4.6 \times 10^{7} \mathrm{MeV}^{-2}, \quad G_{\text {eff }}>2.8 \mathrm{MeV}^{-2}\right.$, or other combinations) where $\Gamma$ is always smaller than $H$. This region exhibits an exotic behavior that suggests that, for the very large thermal mass corrections, neutrinos may not get into thermal equilibrium with the radiation plasma. Avoiding such a nonphysical scenario imposes a natural bound on the thermal mass and, thus, to the couplings. In Fig. 3, we show the numerical results for $T_{\mathrm{dec}}$ in the SNSI parameter grid; there the parameter region where the thermal mass surpasses acceptable values had been excluded.

The neutrino density freezes out at their decoupling temperature, and no significant number of neutrinos gets produced after that. This is because weak interactions would be able to produce only a small percentage of the total neutrino density. Lastly, notice that assuming this late instantaneous neutrino decoupling is the most conservative approach. However, we expect almost the same final neutrino density as using a more complex model for neutrino decoupling, since, in the standard case, neutrinos decouple the earliest at $\sim 3 \mathrm{MeV}$.

We move on to model the neutrino density. The energy density becomes a piecewise function, where the neutrino density freezes out at the threshold $T_{\gamma}=T_{\text {dec }}$ :

$\rho_{\nu}= \begin{cases}\rho_{\nu}\left(G_{\mathrm{eff}}, G_{\mathrm{S}} ; T_{\nu}, T_{\gamma}\right) & T_{\gamma}>T_{\mathrm{dec}} \\ \left(\frac{T_{\nu}}{T_{\mathrm{dec}}}\right)^{4} \rho_{\nu}\left(G_{\mathrm{eff}}, G_{\mathrm{S}} ; T_{\mathrm{dec}}, T_{\mathrm{dec}}\right) & T_{\gamma} \leq T_{\mathrm{dec}}\end{cases}$

where $\rho_{\nu}\left(G_{\text {eff }}, G_{\mathrm{S}} ; T_{\nu}, T_{\gamma}\right)$ is the thermal density described in Eq. (5). After decoupling, the neutrino density falls due to the adiabatic expansion of the Universe. We sketch this in Figs. 4(a) and 4(b) for different values of the SNSI effective parameters.

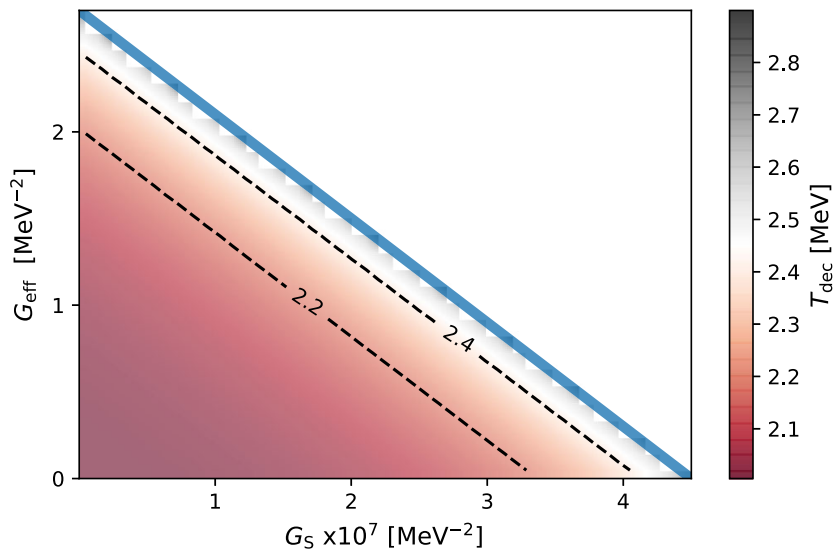

FIG. 3. Neutrino decoupling temperature as a function of the SNSI parameters. The blue line denotes the threshold where the neutrino effective mass becomes too large, spoiling its standard thermalization. 

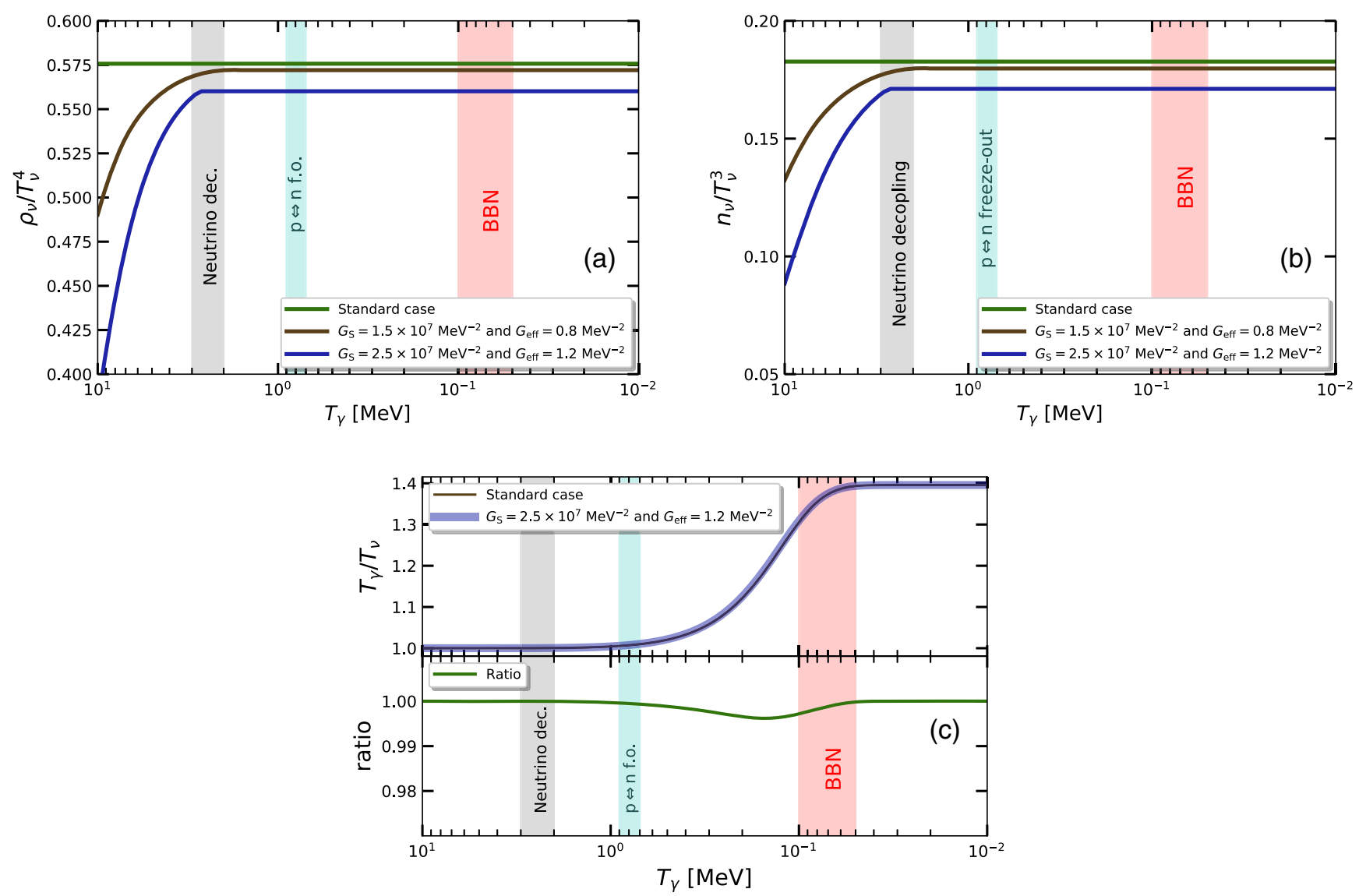

FIG. 4. Neutrino density and temperature evolution for different values of the SNSI effective couplings. Key cosmological events highlighted: neutrino decoupling, proton to neutron freeze-out, and synthesis of light elements. (a) Neutrino energy density evolution. (b) Neutrino number density evolution. (c) Evolution of neutrino temperature with respect to the photon temperature.

We also track the neutrino temperature evolution after decoupling. We employ a modified version of the public available code NUDEC_BSM $[13,45]$. This code solves for the ratio of the neutrino and photon temperature in a much simpler approximation than state-of-the-art codes [53]. Unlike other precise computations of $N_{\text {eff }}$, this code does not include neutrino oscillations, yet it computes a pretty robust value of $N_{\text {eff }}=3.045$ in the SM case. In Fig. 4(c), we depicted the evolution of the temperatures for the standard case and a scenario denoted as large $G_{\text {eff }}$ and $G_{\mathrm{S}}$ values. We observe that the evolution of temperatures differs only within the numerical error values.

We proceed to numerically compute the effective number of relativistic species $N_{\text {eff }}$. For this purpose, we again employ the code NUDEC_BSM. We observe that there is a direct relation between the SNSI parameters and $N_{\text {eff }}$; this is given by

$N_{\text {eff }}\left(G_{\text {eff }}, G_{\mathrm{S}}\right) \equiv \frac{8}{7}\left(\frac{11}{4}\right)^{4 / 3} \frac{3 \rho_{\nu}\left(G_{\mathrm{S}}, G_{\text {eff }}\right)}{\rho_{\gamma}}$ for $T_{\gamma} \ll m_{e}$, where $\rho_{\gamma}=\left(2 \pi^{2} / 30\right) T_{\gamma}^{4}$ is the photon density and we have assumed a full degeneration of neutrino parameters. In Fig. 5, we illustrate the change on $N_{\text {eff }}$ as a function of the effective couplings $G_{\mathrm{S}}$ and $G_{\text {eff }}$, where we used $T_{\nu} / T_{\gamma} \sim$ 0.7164 as obtained from the code NUDEC_BSM. Notice that,

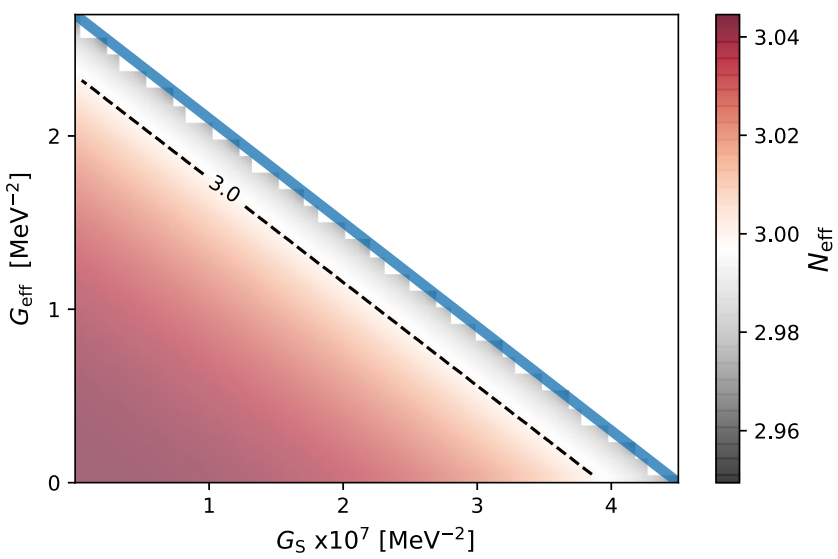

FIG. 5. $N_{\text {eff }}$ as a function of the effective couplings $G_{\mathrm{S}}$ and $G_{\text {eff }}$. The blue line denotes the threshold beyond which the effective mass spoils neutrino standard thermal history. 
in the limit $G_{\mathrm{S}}, G_{\text {eff }} \rightarrow 0$, we recover $N_{\text {eff }} \simeq 3.04$. We can observe a strong positive correlation between the SNSI parameters, since they both produce the same effect. In the next section, we will constrain these parameters with BBN physics.

Lastly, notice that we are neglecting first-order SNSI processes, such as three-level scattering. Thus, we assume that they do not contribute to any change in the neutrino temperature, production, and decoupling. Besides, we assume that the scalar mediator is out of equilibrium with the plasma, so it does not acquire a thermal mass nor is being thermally produced within the plasma at a significant number. Finally, we assume that neutrino decays into the scalar are irrelevant; hence, the scalar density is insignificant.

Dimensional arguments permit us to explore the validity of these approximations. Our region of interest lies in the small scalar mass regime $m_{\phi} \ll \mathrm{keV}$. In this case, the SNSI cross section of processes such as $e^{-} \nu \rightarrow e^{-} \nu$ would be $\sigma_{\mathrm{SNSI}} \approx g_{e}^{2} g_{\nu}^{2} / T^{2}$, while the SM cross section is given by $\sigma_{\mathrm{SM}} \approx \alpha^{2} T^{2} / M_{w}^{4}$, where $\alpha \sim 1 / 137$ is the fine-structure constant and $M_{w} \sim 80 \mathrm{GeV}$ is the $W$ boson mass. Comparing both cross sections, we observed that the condition $g_{e} g_{\nu}<\alpha T^{2} / M_{w}^{2}$, for $T \sim 1 \mathrm{MeV}$ implies that $g_{e} g_{\nu} \lesssim 10^{-12}$. Similarly, the scalar would be prevented from reaching thermal equilibrium as long as the condition $g_{\nu}^{2}<\alpha T^{2} / M_{w}^{2}$ is satisfied. Lastly, the scalar would not significantly contribute to $N_{\text {eff }}$, provided the condition $g_{e}<g_{\nu} \lesssim 10^{-5}$ for $m_{\phi} \ll \mathrm{keV}$ [31] is not violated. As we will argue along Sec. V, these conditions would be satisfied in the ultralight scalar regime (see Figs. 7 and 8).

\section{BBN CONSTRAINTS}

In this section, we present the bounds on the effective SNSI parameters $G_{\mathrm{S}}$ and $G_{\text {eff }}$ by BBN theory and observations of light element abundances.

$\mathrm{BBN}$ is one of the cornerstones of modern cosmology and the big bang theory. With the interplay of standard nuclear and particle physics and the standard cosmological model, it describes with great accuracy the synthesis of the lighter nuclei during the very first seconds of cosmic time (for a review, see [54]). Despite some uncertainties on the predictions for ${ }^{7} \mathrm{Li}$, which may have a diversity of possible sources $[55,56]$, it predicts the observed relative abundances of $\mathrm{H}, \mathrm{D},{ }^{3} \mathrm{He},{ }^{4} \mathrm{He}$, and ${ }^{7} \mathrm{Li}$ as a function of a single parameter, the baryon-to-photon ratio $\eta=n_{b} / n_{\gamma}$, or, equivalently, the present baryon density $\Omega_{b} h^{2}$, which determines the end of the deuterium bottleneck and, therefore, the production rate of heavier nuclei.

Aside from the initial condition on $\eta$, which is thought to be associated with an earlier baryogenesis process, for which the SM seems to have not a satisfactory explanation, BBN success is based on well-known physics, which leaves little space for new or exotic physics. This feature is precisely what makes $\mathrm{BBN}$ a useful probe for any nonstandard physics that may modify the cosmological evolution during those early times, in particular, any physics that could change the expansion rate during BBN [10,57,58]. As the effective neutrino mass that we are discussing changes $N_{\text {eff }}$, it does affect the amount of radiation during that epoch, and so $\mathrm{BBN}$ should be sensitive to it. We will focus on this in what follows.

To a good approximation, when the deuterium bottleneck breaks up, most of the neutrons present in the primordial Universe are synthesized in ${ }^{4} \mathrm{He}$. Other elements are then produced at much smaller amounts, with a rate of about $10^{-5}$ for $\mathrm{D}$ and ${ }^{3} \mathrm{He}$ and $10^{-10}$ for ${ }^{7} \mathrm{Li}$ per proton. ${ }^{4} \mathrm{He}$ mass fraction is well approximated as

$$
Y_{p} \approx \frac{2(n / p)}{1+(n / p)},
$$

where the neutron-to-proton ratio $(n / p)$ at $\mathrm{BBN}$ is determined by the output ratio at weak interaction freeze-out, when the weak interaction rate per baryon $\Gamma_{e w} \approx \alpha^{2} T^{5} / M_{w}^{4}$ becomes smaller than Hubble expansion, and by neutron number depletion due to $\beta$ decay. In the standard cosmological model, at temperatures well above the neutronproton mass difference, $\Delta m=m_{n}-m_{p}=1.239 \mathrm{MeV}$, neutrons and protons are in chemical equilibrium. Below that, temperature electron neutrino capture process $n \nu \rightarrow$ pe starts favoring protons. Since $\eta$ is small, this process does not sensitively alter lepton population, and, thus, the corresponding Boltzmann equation is written as

$$
\frac{d X_{n}}{d t}=\lambda_{n p}\left[\left(1-X_{n}\right) e^{-\Delta m / T}-X_{n}\right]
$$

where $Y_{p}=2 X_{n}$ and $\lambda_{n p}=n_{\nu}^{(0)}\langle\sigma v\rangle$. Note that the last is mostly independent of $n_{\nu}^{(0)}$ but sensitive to the neutrino spectrum, since $\langle\sigma v\rangle=\mathcal{I} /\left(n_{\nu}^{(0)} n_{n}^{(0)}\right)$, with $\mathcal{I}$ an integral over all particle momentum space of the differential cross section weighted by the Boltzmann factor $e^{-\left(E_{\nu}+E_{n}\right) / T}$. At freeze-out temperature $T_{\star} \sim 0.8 \mathrm{MeV},(n / p)_{\star} \simeq e^{-\Delta m T_{\star}} \sim 1 / 5$, and, thus, one estimates $(n / p)_{\mathrm{BBN}} \simeq 1 / 7$ (for a theoretical calculation of this, see, for instance, $[59,60])$.

The key feature for our present analysis resides in the fact that setting $N_{\text {eff }}$ as a free parameter compromises the expansion rate during the radiation-dominated epoch. A smaller (larger) value of $N_{\text {eff }}$ than the one computed in the standard case reduces (increases) expansion rate and lowers (raises) weak interaction decoupling temperature. Even if the change is mild, due to Boltzmann suppression, a smaller (larger) $T_{\star}$ implies a lower (higher) $(n / p)$ and, thus, a smaller (higher) $Y_{p}$. Notice that there is a competing effect when an excess (deficit) of neutrinos over the equilibrium spectrum exists, since it increases (decreases) weak rates, implying a smaller (larger) $Y_{p}$ [61]. However, as effective neutrino thermal mass mainly affects the 
low-energy part of the spectrum, where the differential cross section quickly dies down, the last effect is expected to be less relevant against varying $N_{\text {eff }}$.

CMB is sensitive to both $\eta$ and $Y_{p}$, and, as a matter of fact, Planck data alone provide a determination of $Y_{p}$ [1]. Although $Y_{p}$ is not sensitive to the baryon-to-photon ratio, as we mentioned earlier, $\eta$ is an important initial condition for BBN and the production of other light elements. Here, we keep our analysis consistent with CMB using a prior for $\eta$ consistent with the permitted region at $1 \sigma$ by Planck data $\Omega_{b} h^{2}=0.0224 \pm 0.0001$ or, equivalently, $\eta=6.11 \pm 0.03 \times 10^{-10}$.

In order to constraint the SNSI effective parameters, we use the observations of primordial deuterium and helium abundances. For our purpose, we employ a modified version of the public code ALTERBBN [46,47], where, hereafter, we use a neutron lifetime $\tau_{n}=880.2 \mathrm{~s}$. We use a $\chi^{2}$ analysis with

$$
\chi^{2}=\sum \frac{\left(R_{\mathrm{SNSI}}-R_{\mathrm{obs}}\right)^{2}}{\sigma^{2}},
$$

where $R_{\mathrm{obs}}$ and $R_{\mathrm{SNSI}}$ are, respectively, the observed and theoretical nucleon fractions and $\sigma$ its observational error [62]. The sum is over the two measurements of helium and deuterium fractions, $Y_{p}=0.245 \pm 0.003$ and $\mathrm{D} / \mathrm{H}=$ $(2.569 \pm 0.027) \times 10^{-5}$. In Fig. 6 (lhs), we present the fluctuation of the $\chi^{2}$ function $\Delta \chi^{2}=\chi^{2}-\chi_{\min }^{2}$ as a function of the parameter $N_{\text {eff. }}$. Notice that the $\chi^{2}$ is not symmetric and gets steeper for small values of $N_{\text {eff }}$. Therefore, this lets us set stringent constraints to the SNSI parameters.

As we mentioned earlier, we obtain the constraints on the SNSI effective parameters by taking advantage of the direct relation between $N_{\text {eff }}$ and a pair $G_{\mathrm{S}}$ and $G_{\text {eff }}$ (see Fig. 5). In Fig. 6 (rhs), we present the deviation of $\chi^{2}$ from its minimum value as a function of the SNSI parameters. Notice that we have two strongly correlated parameters; thus, we employ a statistical procedure in which we can find a robust bound for each parameter.

In order to find the bounds for $G_{\text {eff }}$ and $G_{S}$, we use the posterior distribution $P\left(\theta_{1}, \theta_{2}, \eta\right) \propto e^{-\chi^{2} / 2}$, where $\theta_{1}$ is the parameter that we are analyzing, either $G_{\text {eff }}$ or $G_{\mathrm{S}}$. We marginalize over the second parameter $\theta_{2}$ and the baryonto-photon ratio $\eta$ to obtain the single-parameter posterior distribution $P^{(1)}\left(\theta_{1}\right) \propto \iint P\left(\theta_{1}, \theta_{2}\right) d \theta_{2} d \eta$. Then we follow Ref. [63] to find the credible intervals for our situation. In our case, the minimum of the credible intervals coincides with the physical bound of the parameters $G_{\mathrm{S}}$ and $G_{\text {eff }}$ which are constrained to be positive. The $100 \gamma \%$ credible region will be defined as

$$
\int_{0}^{\theta_{1 \mathrm{bound}}} d \theta_{1} P^{(1)}\left(\theta_{1}\right)=\gamma_{1} \text {. }
$$

Using a finite grid, the $\gamma$ value for the parameter $\theta_{1}$ is

$$
\gamma_{1}=\frac{1}{N} \sum_{i=0}^{i_{\text {bound }}} \sum_{j, k} \mathrm{e}^{-\chi_{i j k}^{2} / 2} \delta \theta_{1 i} \delta \theta_{2 j} \delta \eta_{j},
$$

where $N=\sum_{i, j, k} \mathrm{e}^{-\chi_{i j k}^{2} / 2} \delta \theta_{1 i} \delta \theta_{2 j} \delta \eta_{j}, \delta \theta$ is a small constant finite difference in the parameter sampling, and $\delta \eta$ is similarly defined.

We obtain the parameter constraints at $68 \%$ C.L. by finding the $\theta$ values that make $\gamma_{1}=\gamma_{2}=0.68$. Our final marginalized bounds are

$$
\begin{aligned}
G_{\text {eff }} & <1.2 \mathrm{MeV}^{-2} \quad(68 \% \text { C.L. }) . \\
G_{\mathrm{S}} & <2.0 \times 10^{7} \mathrm{MeV}^{-2} \quad(68 \% \text { C.L. }) .
\end{aligned}
$$

Notice that, although parameter marginalization is the appropriate statistical procedure to obtain robust constraints, the effective SNSI parameters are strongly

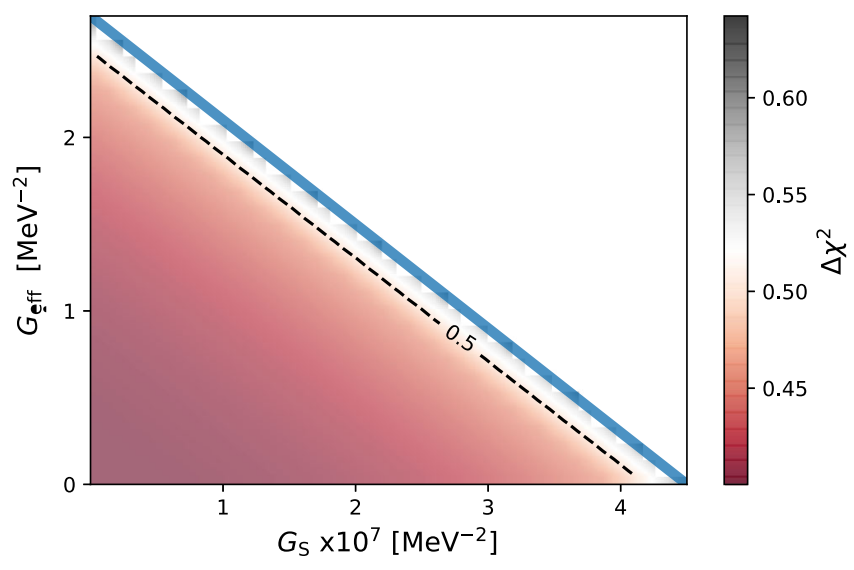

FIG. 6. $\chi^{2}$ as a function of the model parameters with $\eta=6.11 \times 10^{-10}$. Left panel: $\Delta \chi^{2}$ as a function of the effective number of relativistic species $N_{\text {eff }}$. Measurements of helium- 4 and deuterium. Right panel: $\Delta \chi^{2}$ as a function of the effective couplings $G_{\mathrm{S}}$ and $G_{\text {eff }}$. As before, the blue line denotes the threshold above which neutrino standard thermalization gets spoiled. 
positively correlated and the degeneracy cannot be entirely broken up.

In the next section, we will disentangle the model parameters (the couplings and the mediator mass) by using our constraints in the effective SNSI parameters and present new bounds on the neutrino-scalar coupling.

\section{PARAMETER SPACE COMPARISON}

We have discussed and computed the bounds on the effective parameters of SNSI. Here we translate the bounds into the mass-coupling parameter space and compare our constraints with others from terrestrial experiments as well as astrophysical and cosmological observations.

First, we should discuss one important limit in the cosmological approach. As stated by Ref. [41], the scalar mass has a lower bound imposed by the size of the Universe at the relevant epochs. This is because the de Broglie wavelength of the particle, $l \propto m_{\phi}^{-1}$, cannot be larger than the size of the Universe. Otherwise, it will escape the Hubble horizon. For our considerations, we establish the scalar mass lower bound from Hubble radius at $2 \mathrm{MeV}, H^{-1}(2 \mathrm{MeV})$. Thus, our results are valid only for $m_{\phi} \gtrsim 1.5 \times 10^{-15} \mathrm{eV}$. Interestingly, notice that the size of the Hubble horizon at those epochs is smaller than the size of the Sun.

Here, we present a new stringent bound on the scalarneutrino coupling $g_{\nu}$, which is particularly robust for ultralight scalar masses. Note that the bound on $G_{\mathrm{S}}$ [from Eq. (15)] permits us to find a mass-dependent bound on $g_{\nu}$, that goes as

$$
g_{\nu}<4.5 \times 10^{-3}\left(\frac{m_{\phi}}{\mathrm{eV}}\right) \quad(68 \% \text { C.L. }) .
$$

This new bound restricts a large new region in the parameter space $\left(m_{\phi}, g_{\nu}\right)$ for masses $1.5 \times 10^{-15} \mathrm{eV} \lesssim$ $m_{\phi} \lesssim 4.5 \times 10^{-5} \mathrm{eV}$, where the upper value comes from $g_{\nu}<2 \times 10^{-7}$ derived by the authors in Ref. [35].

In the literature, we spot that there have been extensive efforts to impose bounds on the neutrino-scalar coupling. For instance, it has been constrained by coherent elastic neutrino-nucleus scattering $(\mathrm{CE} \nu \mathrm{NS})$ and by scalar emission in neutrinoless double-beta decay experiments $[30,32,33,64]$. Interestingly, a neutrino-scalar coupling around $\sim 10^{-6}$ could explain the recent anomalous spectral excess at the XENONvT experiment $[65,66]$.

Astrophysical and cosmological observations set the strongest constraints on the neutrino-scalar coupling. A neutrino flavor-dependent scalar interaction is responsible for several nonobserved effects in supernovas (SN). Such effects include a loss of SN luminosity, loss of leptons in the supernova core (deleptonization), and trapping of neutrinos by dispersion with a (pseudo)scalar. In Fig. 7, we depict the strongest $\mathrm{SN}$ bounds, corresponding to a (pseudo)scalar coupled to electron neutrinos $\left|g_{\text {ee }}\right|[29,33]$.

We also revisit a couple of cosmological bounds. The bound imposed by Ref. [31] confronts the positive contribution of a light scalar particle to $N_{\text {eff }}$, namely, $\Delta N_{\text {eff }}$, with BBN physics. On the other hand, in Refs. [34,35], the authors studied the observable effects on the CMB caused by neutrino self-interactions mediated by a very light scalar particle $m_{\phi} \ll T_{\nu}$. As neutrinos become collisional again at small temperatures, this approximation holds for $m_{\phi} \ll T_{\nu}(z=100)$, roughly $m_{\phi} \lesssim 10^{-3} \mathrm{eV}$. They found the bound $g_{\nu \text {,eff }}<2 \times 10^{-7}$, where the ratio between $g_{\nu \text {,eff }}$ and $g_{\nu}$ is no larger than one order of magnitude. Finally, in the scalar mass region $10^{-1} \mathrm{eV} \lesssim m_{\phi} \lesssim 10^{3} \mathrm{eV}$ and using CMB data, a fairly robust neutrino-scalar bound was found by Escudero and Witte [67]. In Fig. 7, we depicted all these bounds including our new measurement.

We now discuss the bound on the electron-scalar coupling $g_{e}$. Notice that here we cannot set a direct constraint to $g_{e}$, because we do not have a direct measurement on $g_{\nu}$; we have only an upper bound. We can only estimate where the bound would lie by using the constraint on $G_{\mathrm{S}}$ together with the bound on $G_{\text {eff }}$ from Eq. (15). Taking the $g_{\nu}$ upper value given in Eq. (16), we estimate

$$
g_{e}<2.7 \times 10^{-10}\left(\frac{m_{\phi}}{\mathrm{eV}}\right) .
$$

Supposing an eventual future measurement of $g_{\nu}$, we observe that the bound on $g_{e}$ is weaker in our analysis than those obtained from neutrinos SNSI from the Sun and SN. The Sun bound is particularly interesting, since Ref. [42]

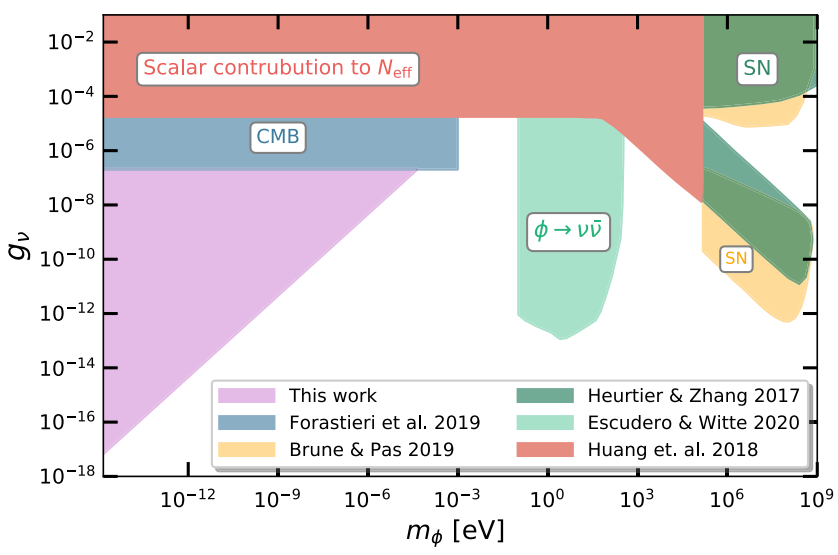

FIG. 7. Neutrino-scalar coupling constraints. The purple area represents the region excluded by our bound on $G_{\mathrm{S}}<$ $2.0 \times 10^{7} \mathrm{MeV}^{-2}$. The blue area represents the $\mathrm{CMB}$ excluded region for scalar-mediated self-interacting neutrinos [35]. The turquoise area represents the $\mathrm{CMB}$ constraints by scalar decays into neutrinos [67]. The red area represents the excluded region for a scalar contributing to $N_{\text {eff }}$ during BBN [31]. Green and orange areas portray the region excluded by supernovae $[29,33]$. 


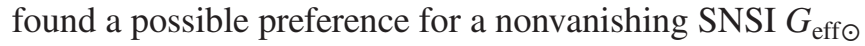
effective coupling. As a matter of fact, aside from the fluctuation, we can safely take the solar bound as $\Delta m_{\odot}<7.4 \times 10^{-3} \mathrm{eV}$. The solar medium is nonrelativistic; therefore, the mass correction in the Sun goes as $\Delta m_{\odot}=G_{\text {eff } \odot} n_{\mathrm{e} \odot}$, where the number density of electrons at the solar core is $n_{\mathrm{e} \odot} \sim 5.2 \times 10^{11} \mathrm{eV}^{3}$. With this, $g_{e}=m_{\phi}^{2} \Delta m_{\odot} / g_{\nu}$. Comparing the solar bound on $\Delta m_{\odot}$ with our early Universe bound, and taking the upper value in Eq. (16), we observe that, indeed, the early Universe bound is weaker than the solar one (see Fig. 8).

In Fig. 8, we show different constraints compared with our results. On the one hand, there are strong bounds, $g_{e}<10^{-15}$, from stellar physics where an electron-scalar coupling would diminish stars to a cooler than expected state. This is due to energy loss caused by the unopposed escape of scalar particles produced from the stellar nucleus $[68,69]$. On the other hand, fifth-force experiments that search for deviations to the Newtonian gravity set the strongest bounds for electron-scalar coupling with a very light mediator. The length scale of the gravitational experiments is related to the force mediator mass, which constraints can be directly obtained from Ref. [70]. Experimental constraints at shorter lengths were reviewed and summarized by Ref. [41] with the results of several experiments [71]. Here, we depict the compendium of fifth-force experiments within a single bound in Fig. 8. Additionally, we include the curves indicating the reference bound on $g_{e}$ for a fixed $g_{\nu}$ value in the case of neutrinos propagating in supernovas and the Sun from Ref. [41].

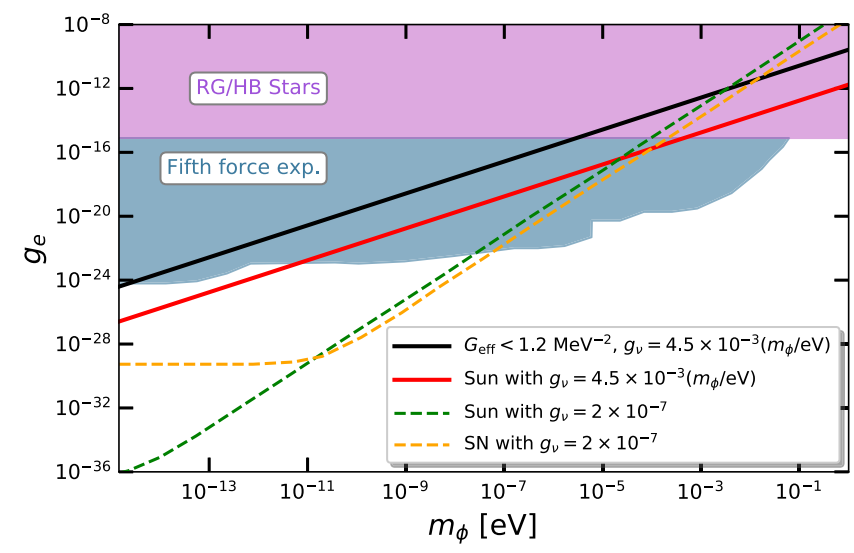

FIG. 8. Electron-scalar coupling constraints. The region that lies above the black line is not permitted by our constraints for a $g_{\nu}$ value fixed to its upper bound. The region above the red line would be prohibited for a $g_{\nu}$ value fixed to its upper bound and the solar neutrino constraint. The purple area represents the region excluded by energy loss in stars. The blue area represents the region prohibited by fifth-force experiments. Dashed lines: SN and Sun bounds by Babu, Chauhan, and Dev [41] for $g_{\nu}=2 \times 10^{-7}$.

\section{SUMMARY AND CONCLUSIONS}

We have performed a robust analysis of the consequences of a possible large neutrino effective mass due to thermal corrections mediated by nonstandard light scalar interactions among leptons in the context of the early Universe. Such an effective mass is fed by CFS of propagating neutrinos through a thermal bath of neutrinos and electrons or positrons within the primordial plasma. At one-loop order, the effective neutrino mass is simply proportional to the respective scalar to neutrino or electron couplings but inversely proportional to the square scalar mass. One can encode such dependencies in a couple of SNSI parameters, $G_{\mathrm{S}}$ and $G_{\text {eff }}$. The effective neutrino mass also depends on the temperature of the corresponding thermal bath, through a monotonically increasing function, such that the higher the temperatures, the larger the effective mass contributions. Hence, even if no visible effects appear at small redshifts, possible changes on standard physics could arise as we look toward earlier times.

In the case where the neutrino effective mass gets comparable with the neutrino temperature, their number and energy density drops significantly. However, the SNSI effect vanishes faster than the temperature drop, and, in equilibrium, the standard neutrino density is recovered. Nevertheless, once neutrinos decouple from the primordial plasma, its production gets largely suppressed; thus, their density at decoupling freezes out. This has an observable direct effect that is expressed as a smaller $N_{\text {eff }}$ than expected.

BBN has shown to be sensitive to any nonstandard physics that affects the expansion rate. We have exploited this feature and used BBN primordial nuclei outputs and observational data to set a constraint on the neutrino-scalar coupling. Our new bound on $g_{\nu}$ is more restrictive that previously known bounds for the mass range $1.5 \times 10^{-15} \mathrm{eV} \lesssim m_{\phi} \lesssim 4.5 \times 10^{-5} \mathrm{eV}$.

Although our analysis is able to constrain the scalarelectron couplings, it does also involve scalar-neutrino coupling, and, thus, no straightforward bound to the former can be set without knowledge about the latter. Nevertheless, we have explored the parameter space assuming the saturation of our bound on the scalar-neutrino coupling to compare with other results from astrophysics and fifthforce experimental limits.

Along with our analysis, we have assumed that the light scalar mediator would play no direct role in early cosmology, by looking upon the parameter range where it would stay out of equilibrium and its production rate suppressed during the early Universe. In the opposite scenario, neutrino NSI may have other consequences that can be further studied in cosmology. For instance, neutrino NSI may trigger active neutrino decays and annihilation into light bosons. Adding such effects to our analysis would probably amount to softening our bounds, since light 
scalars add to the relativistic degrees of freedom, raising $N_{\text {eff }}$, and compensating the effect of thermal neutrino mass $[31,72]$. Furthermore, neutrino decay and annihilation during the structure formation era could relax the bound on $\Sigma m_{\nu}$ from LSS [36-40]; in contrast, larger bare neutrino masses impose more stringent constraints. Such analysis may be worthy of being pursued.

The physics of nonstandard neutrino interactions is an active field of study due to its potential to solve current tensions in cosmology. The early Universe can be used as a testing ground to study such interactions in environments unreachable by terrestrial or solar experiments. In this work, we used the indirect effect of neutrinos on the relic densities of light elements to impose bounds upon the possible interactions with a light scalar mediator. This bound is stronger than the previous bounds and contributes to a better understanding of the nature of neutrinos and its possible links to physics outside the standard model of particles.

\section{ACKNOWLEDGMENTS}

This work has been partially supported by Conacyt, Mexico, under FORDECYT-PRONACES Grant No. 490769. We thank two anonymous referees for their critical reviews that led to an improvement of our paper.
[1] N. Aghanim et al. (Planck Collaboration), Planck 2018 results-vi. Cosmological parameters, Astron. Astrophys. 641, A6 (2020).

[2] N. Palanque-Delabrouille et al., Neutrino masses and cosmology with lyman-alpha forest power spectrum, J. Cosmol. Astropart. Phys. 11 (2015) 011.

[3] A. Loureiro et al., Upper Bound of Neutrino Masses from Combined Cosmological Observations and Particle Physics Experiments, Phys. Rev. Lett. 123, 081301 (2019).

[4] S. Gariazzo, M. Archidiacono, P. de Salas, O. Mena, C. Ternes, and M. Tórtola, Neutrino masses and their ordering: Global data, priors and models, J. Cosmol. Astropart. Phys. 03 (2018) 011.

[5] M. Aker et al. (KATRIN Collaboration), Improved Upper Limit on the Neutrino Mass from a Direct Kinematic Method by Katrin, Phys. Rev. Lett. 123, 221802 (2019).

[6] T. Brinckmann, D. C. Hooper, M. Archidiacono, J. Lesgourgues, and T. Sprenger, The promising future of a robust cosmological neutrino mass measurement, J. Cosmol. Astropart. Phys. 01 (2019) 059.

[7] M. Archidiacono, S. Hannestad, and J. Lesgourgues, What will it take to measure individual neutrino mass states using cosmology?, J. Cosmol. Astropart. Phys. 09 (2020) 021.

[8] R. J. Cooke, M. Pettini, and C. C. Steidel, One percent determination of the primordial deuterium abundance, Astrophys. J. 855, 102 (2018).

[9] E. Aver, K. A. Olive, and E. D. Skillman, The effects of he i $\lambda 10830$ on helium abundance determinations, J. Cosmol. Astropart. Phys. 07 (2015) 011.

[10] A. Peimbert, M. Peimbert, and V. Luridiana, The primordial helium abundance and the number of neutrino families, Rev. Mexicana Astron. Astrofísica 52, 419 (2016).

[11] G. Mangano, G. Miele, S. Pastor, T. Pinto, O. Pisanti, and P. D. Serpico, Relic neutrino decoupling including flavour oscillations, Nucl. Phys. B729, 221 (2005).

[12] P. F. de Salas and S. Pastor, Relic neutrino decoupling with flavour oscillations revisited, J. Cosmol. Astropart. Phys. 07 (2016) 051.
[13] M.E. Abenza, Precision early universe thermodynamics made simple: Neff and neutrino decoupling in the standard model and beyond, J. Cosmol. Astropart. Phys. 05 (2020) 048 .

[14] K. Akita and M. Yamaguchi, A precision calculation of relic neutrino decoupling, J. Cosmol. Astropart. Phys. 08 (2020) 012.

[15] J. Froustey, C. Pitrou, and M. C. Volpe, Neutrino decoupling including flavour oscillations and primordial nucleosynthesis, J. Cosmol. Astropart. Phys. 12 (2020) 015.

[16] S. Bashinsky and U. Seljak, Signatures of relativistic neutrinos in cmb anisotropy and matter clustering, Phys. Rev. D 69, 083002 (2004).

[17] B. Follin, L. Knox, M. Millea, and Z. Pan, First Detection of the Acoustic Oscillation Phase Shift Expected from the Cosmic Neutrino Background, Phys. Rev. Lett. 115, 091301 (2015).

[18] D. Baumann, F. Beutler, R. Flauger, D. Green, A. Slosar, M. Vargas-Magaña, B. Wallisch, and C. Yèche, First constraint on the neutrino-induced phase shift in the spectrum of baryon acoustic oscillations, Nat. Phys. 15, 465 (2019).

[19] L. Wolfenstein, Neutrino oscillations and stellar collapse, Phys. Rev. D 20, 2634 (1979).

[20] S. N. Ahmed et al. (SNO Collaboration), Measurement of the Total Active ${ }^{8} \mathrm{~B}$ Solar Neutrino Flux at the Sudbury Neutrino Observatory with Enhanced Neutral Current Sensitivity, Phys. Rev. Lett. 92, 181301 (2004).

[21] C. Arpesella et al. (Borexino Collaboration), Direct Measurement of the ${ }^{7} \mathrm{Be}$ Solar Neutrino Flux with 192 Days of Borexino Data, Phys. Rev. Lett. 101, 091302 (2008).

[22] M. A. Acero et al. (NOvA Collaboration), First Measurement of Neutrino Oscillation Parameters Using Neutrinos and Antineutrinos by Nova, Phys. Rev. Lett. 123, 151803 (2019).

[23] K. Abe et al. (The T2K Collaboration), Search for Electron Antineutrino Appearance in a Long-Baseline Muon Antineutrino Beam, Phys. Rev. Lett. 124, 161802 (2020).

[24] K. C. Wong et al. (H0liCOW Collaboration), H0licow xiii. A $2.4 \%$ measurement of $\mathrm{H} 0$ from lensed quasars: $5.3 \sigma$ 
tension between early and late-universe probes, Mon. Not. R. Astron. Soc. 498, 1420 (2020).

[25] A. G. Riess, S. Casertano, W. Yuan, L. M. Macri, and D. Scolnic, Large magellanic cloud cepheid standards provide a $1 \%$ foundation for the determination of the hubble constant and stronger evidence for physics beyond $\Lambda \mathrm{CDM}$, Astrophys. J. 876, 85 (2019).

[26] L. Verde, T. Treu, and A. G. Riess, Tensions between the early and late universe, Nat. Astron. 3, 891 (2019).

[27] S. Hannestad, R. S. Hansen, and T. Tram, How SelfInteractions can Reconcile Sterile Neutrinos with Cosmology, Phys. Rev. Lett. 112, 031802 (2014); B. Dasgupta and J. Kopp, Cosmologically Safe ev-Scale Sterile Neutrinos and Improved Dark Matter Structure, Phys. Rev. Lett. 112, 031803 (2014); M. Archidiacono, S. Hannestad, R. S. Hansen, and T. Tram, Cosmology with self-interacting sterile neutrinos and dark matter: A pseudoscalar model, Phys. Rev. D 91, 065021 (2015); M. Archidiacono, S. Gariazzo, C. Giunti, S. Hannestad, R. Hansen, M. Laveder, and T. Tram, Pseudoscalar-Sterile neutrino interactions: Reconciling the cosmos with neutrino oscillations, J. Cosmol. Astropart. Phys. 08 (2016) 067; M. Archidiacono, S. Hannestad, R. S. Hansen, and T. Tram, Sterile neutrinos with pseudoscalar self-interactions and cosmology, Phys. Rev. D 93, 045004 (2016); X. Chu, B. Dasgupta, M. Dentler, J. Kopp, and N. Saviano, Sterile neutrinos with secret interactions-cosmological discord?, J. Cosmol. Astropart. Phys. 11 (2018) 049; M. Archidiacono, S. Gariazzo, C. Giunti, S. Hannestad, and T. Tram, Sterile neutrino self-interactions: $\mathrm{H} 0$ tension and short-baseline anomalies, J. Cosmol. Astropart. Phys. 12 (2020) 029.

[28] N. F. Bell, E. Pierpaoli, and K. Sigurdson, Cosmological signatures of interacting neutrinos, Phys. Rev. D 73, 063523 (2006); M. Archidiacono and S. Hannestad, Updated constraints on non-standard neutrino interactions from planck, J. Cosmol. Astropart. Phys. 07 (2014) 046; F.-Y. Cyr-Racine and K. Sigurdson, Limits on neutrino-neutrino scattering in the early universe, Phys. Rev. D 90, 123533 (2014); I. M. Oldengott, C. Rampf, and Y. Y. Wong, Boltzmann hierarchy for interacting neutrinos. i: Formalism, J. Cosmol. Astropart. Phys. 04 (2015) 016; L. Lancaster, F.-Y. Cyr-Racine, L. Knox, and Z. Pan, A tale of two modes: Neutrino free-streaming in the early universe, J. Cosmol. Astropart. Phys. 07 (2017) 033; I. M. Oldengott, T. Tram, C. Rampf, and Y. Y. Wong, Interacting neutrinos in cosmology: Exact description and constraints, J. Cosmol. Astropart. Phys. 11 (2017) 027; C. D. Kreisch, F.-Y. CyrRacine, and O. Doré, Neutrino puzzle: Anomalies, interactions, and cosmological tensions, Phys. Rev. D 101, 123505 (2020).

[29] L. Heurtier and Y. Zhang, Supernova constraints on massive (pseudo)scalar coupling to neutrinos, J. Cosmol. Astropart. Phys. 02 (2017) 042.

[30] Y. Farzan, M. Lindner, W. Rodejohann, and X.-J. Xu, Probing neutrino coupling to a light scalar with coherent neutrino scattering, J. High Energy Phys. 05 (2018) 66.

[31] G.-y. Huang, T. Ohlsson, and S. Zhou, Observational constraints on secret neutrino interactions from big bang nucleosynthesis, Phys. Rev. D 97, 075009 (2018).
[32] N. Blinov, K. J. Kelly, G. Krnjaic, and S. D. McDermott, Constraining the Self-Interacting Neutrino Interpretation of the Hubble Tension, Phys. Rev. Lett. 123, 191102 (2019).

[33] T. Brune and H. Päs, Massive Majorons and constraints on the Majoron-neutrino coupling, Phys. Rev. D 99, 096005 (2019).

[34] F. Forastieri, M. Lattanzi, and P. Natoli, Constraints on secret neutrino interactions after planck, J. Cosmol. Astropart. Phys. 07 (2015) 014.

[35] F. Forastieri, M. Lattanzi, and P. Natoli, Cosmological constraints on neutrino self-interactions with a light mediator, Phys. Rev. D 100, 103526 (2019).

[36] J. F. Beacom, N. F. Bell, and S. Dodelson, Neutrinoless Universe, Phys. Rev. Lett. 93, 121302 (2004).

[37] S. Hannestad, Structure formation with strongly interacting neutrinos-implications for the cosmological neutrino mass bound, J. Cosmol. Astropart. Phys. 02 (2005) 011.

[38] M. Escudero and M. Fairbairn, Cosmological constraints on invisible neutrino decays revisited, Phys. Rev. D 100, 103531 (2019).

[39] Z. Chacko, A. Dev, P. Du, V. Poulin, and Y. Tsai, Cosmological limits on the neutrino mass and lifetime, J. High Energy Phys. 04 (2020) 20.

[40] M. Escudero, J. Lopez-Pavon, N. Rius, and S. Sandner, Relaxing cosmological neutrino mass bounds with unstable neutrinos, J. High Energy Phys. 12 (2020) 119.

[41] K. S. Babu, G. Chauhan, and P. S. B. Dev, Neutrino nonstandard interactions via light scalars in the earth, sun, supernovae, and the early universe, Phys. Rev. D 101, 095029 (2020).

[42] S.-F. Ge and S. J. Parke, Scalar Nonstandard Interactions in Neutrino Oscillation, Phys. Rev. Lett. 122, 211801 (2019).

[43] A. Y. Smirnov and X.-J. Xu, Wolfenstein potentials for neutrinos induced by ultra-light mediators, J. High Energy Phys. 12 (2019) 46.

[44] A. N. Khan, W. Rodejohann, and X.-J. Xu, Borexino and general neutrino interactions, Phys. Rev. D 101, 055047 (2020).

[45] M. Escudero, Neutrino decoupling beyond the standard model: CMB constraints on the dark matter mass with a fast and precise Neff evaluation, J. Cosmol. Astropart. Phys. 02 (2019) 007.

[46] A. Arbey, AlterbBn: A program for calculating the bbn abundances of the elements in alternative cosmologies, Comput. Phys. Commun. 183, 1822 (2012).

[47] A. Arbey, J. Auffinger, K. Hickerson, and E. Jenssen, ALTERBBN v2: A public code for calculating big-bang nucleosynthesis constraints in alternative cosmologies, Comput. Phys. Commun. 248, 106982 (2020).

[48] J. Liu, Neutrino coherent forward scattering and its index of refraction, Phys. Rev. D 45, 1428 (1992).

[49] J. I. Peltoniemi and J. T. Peltoniemi, Coherence conditions for the forward scattering of neutrinos, J. High Energy Phys. 08 (1999) 008.

[50] L. C. Thomas, T. Dezen, E. B. Grohs, and C. T. Kishimoto, Electron-positron annihilation freeze-out in the early universe, Phys. Rev. D 101, 063507 (2020).

[51] V. Mukhanov, Physical Foundations of Cosmology (Cambridge University Press, Cambridge, England, 2005). 
[52] W. Greiner, B. Müller et al., Gauge Theory of Weak Interactions, Vol. 5 (Springer, New York, 1996).

[53] P. F. de Salas and S. Pastor, Relic neutrino decoupling with flavour oscillations revisited, J. Cosmol. Astropart. Phys. 07 (2016) 051.

[54] R. H. Cyburt, B. D. Fields, K. A. Olive, and T.-H. Yeh, Big bang nucleosynthesis: Present status, Rev. Mod. Phys. 88, 015004 (2016).

[55] B. D. Fields, The primordial lithium problem, Annu. Rev. Nucl. Part. Sci. 61, 47 (2011).

[56] S. Starrfield, M. Bose, C. Iliadis, W. R. Hix, C. E. Woodward, and R. M. Wagner, Carbon-oxygen classical novae are galactic ${ }^{7} \mathrm{Li}$ producers as well as potential supernova ia progenitors, Astrophys. J. 895, 70 (2020).

[57] A. Laguë and J. Meyers, Prospects and limitations for constraining light relics with primordial abundance measurements, Phys. Rev. D 101, 043509 (2020).

[58] R. Foot and R. R. Volkas, How neutrino oscillations can induce an effective neutrino number of less than three during big bang nucleosynthesis, Phys. Rev. D 56, 6653 (1997).

[59] J. Bernstein, L. Brown, and G. Feinberg, Cosmological helium production simplified, Rev. Mod. Phys. 61, 25 (1989).

[60] V. Mukhanov, Nucleosynthesis without computer, Int. J. Theor. Phys. 43, 669 (2004).

[61] A. D. Dolgov, Cosmological implications of neutrinos, Surv. High Energy Phys. 17, 91 (2002).

[62] M. Tanabashi et al. (Particle Data Group), Review of particle physics, Phys. Rev. D 98, 030001 (2018).

[63] J. Hamann, S. Hannestad, G. G. Raffelt, and Y. Y. Y. Wong, Observational bounds on the cosmic radiation density, J. Cosmol. Astropart. Phys. 08 (2007) 021.

[64] P. S. Pasquini and O. L. G. Peres, Bounds on neutrino-scalar Yukawa coupling, Phys. Rev. D 93, 053007 (2016).

[65] A. N. Khan, Can nonstandard neutrino interactions explain the XENON1T spectral excess?, Phys. Lett. B 809, 135782 (2020).
[66] E. Aprile et al. (XENON Collaboration), Excess electronic recoil events in XENON1T, Phys. Rev. D 102, 072004 (2020).

[67] M. Escudero and S. J. Witte, A CMB search for the neutrino mass mechanism and its relation to the Hubble tension, Eur. Phys. J. C 80, 294 (2020).

[68] E. Hardy and R. Lasenby, Stellar cooling bounds on new light particles: Plasma mixing effects, J. High Energy Phys. 02 (2017) 33.

[69] S. Knapen, T. Lin, and K. M. Zurek, Light dark matter: Models and constraints, Phys. Rev. D 96, 115021 (2017).

[70] E. G. Adelberger, J. H. Gundlach, B. R. Heckel, S. Hoedl, and S. Schlamminger, Torsion balance experiments: A lowenergy frontier of particle physics, Prog. Part. Nucl. Phys. 62, 102 (2009).

[71] J. K. Hoskins, R. D. Newman, R. Spero, and J. Schultz, Experimental tests of the gravitational inverse-square law for mass separations from 2 to $105 \mathrm{~cm}$, Phys. Rev. D 32, 3084 (1985); J. C. Long, H. W. Chan, A. B. Churnside, E. A. Gulbis, M. C. M. Varney, and J. C. Price, Upper limits to submillimetre-range forces from extra space-time dimensions, Nature (London) 421, 922 (2003); D. J. Kapner, T. S. Cook, E. G. Adelberger, J. H. Gundlach, B. R. Heckel, C. D. Hoyle, and H.E. Swanson, Tests of the Gravitational Inverse-Square Law Below the Dark-Energy Length Scale, Phys. Rev. Lett. 98, 021101 (2007); A. A. Geraci, S. J. Smullin, D. M. Weld, J. Chiaverini, and A. Kapitulnik, Improved constraints on non-Newtonian forces at $10 \mathrm{mi}-$ crons, Phys. Rev. D 78, 022002 (2008); S. Q. Yang, B. F. Zhan, Q. L. Wang, C. G. Shao, L. C. Tu, W. H. Tan, and J. Luo, Test of the gravitational inverse square law at millimeter ranges, Phys. Rev. Lett. 108, 081101 (2012); W.-H. Tan, S.-Q. Yang, C.-G. Shao, J. Li, A.-B. Du, B.-F. Zhan, Q.-L. Wang, P.-S. Luo, L.-C. Tu, and J. Luo, New Test of the Gravitational Inverse-Square Law at the Submillimeter Range with Dual Modulation and Compensation, Phys. Rev. Lett. 116, 131101 (2016).

[72] X. Luo, W. Rodejohann, and X.-J. Xu, Dirac neutrinos and Neff, J. Cosmol. Astropart. Phys. 06 (2020) 058. 